\title{
The nature of the dwarf starforming galaxy associated with GRB 060218/SN 2006aj ${ }^{\star}$
}

\author{
K. Wiersema ${ }^{1}$, S. Savaglio ${ }^{2}$, P. M. Vreeswijk ${ }^{3,4}$, S. L. Ellison ${ }^{5}$, C. Ledoux ${ }^{3}$, S.-C. Yoon ${ }^{1}$, P. Møller ${ }^{6}$, J. Sollerman ${ }^{7}$, \\ J. P. U. Fynbo ${ }^{7}$, E. Pian ${ }^{8}$, R. L. C. Starling ${ }^{1}$, and R. A. M. J. Wijers ${ }^{1}$
}

\author{
1 Astronomical Institute "Anton Pannekoek", University of Amsterdam, Kruislaan 403, 1098 SJ Amsterdam, The Netherlands \\ e-mail: kwrsema@science.uva.nl \\ 2 Department of Physics and Astronomy, Johns Hopkins University, Baltimore, MD 21218, USA \\ 3 European Southern Observatory, Alonso de Córdova 3107, Casilla 19001, Santiago 19, Chile \\ 4 Departamento de Astronomía, Universidad de Chile, Casilla 36-D, Santiago, Chile \\ 5 Department of Physics and Astronomy, University of Victoria, Elliott Building, 3800 Finnerty Rd., Victoria, BC, V8P 1A1, Canada \\ ${ }^{6}$ European Southern Observatory, Karl-Schwarzschild-Strasse 2, 85748 Garching bei München, Germany \\ 7 Dark Cosmology Centre, Niels Bohr Institute, University of Copenhagen, Juliane Maries Vej 30, 2100 Copenhagen, Denmark \\ 8 INAF, Astronomical Observatory of Trieste, 34131 Trieste, Italy
}

Received 18 August 2006 / Accepted 19 December 2006

\section{ABSTRACT}

\begin{abstract}
Context. We present high resolution VLT UVES and low resolution FORS optical spectroscopy of supernova 2006aj and its host galaxy, associated with the nearby $(z=0.03342)$ gamma-ray burst GRB 060218. This host galaxy is a unique case, as it is one of the few nearby GRB host galaxies known, and it is only the second time high resolution spectra have been taken of a nearby GRB host galaxy (after GRB 980425).

Aims. The resolution, wavelength range and S/N of the UVES spectrum combined with low resolution FORS spectra allow a detailed analysis of the circumburst and host galaxy environments.

Methods. We analyse the emission and absorption lines in the spectrum, combining the high resolution UVES spectrum with low resolution FORS spectra and find the metallicity and chemical abundances in the host. We probe the geometry of the host by studying the emission line profiles.

Results. Our spectral analysis shows that the star forming region in the host is metal poor with $12+\log (\mathrm{O} / \mathrm{H})=7.54_{-0.10}^{+0.17}\left(\sim 0.07 Z_{\odot}\right)$, placing it among the most metal deficient subset of emission-line galaxies. It is also the lowest metallicity found so far for a GRB host from an emission-line analysis. Given the stellar mass of the galaxy of $\sim 10^{7} M_{\odot}$ and the $S F R_{\mathrm{H} x}=0.065 \pm 0.005 M_{\odot} \mathrm{yr}^{-1}$, the high specific star formation rate indicates an age for the galaxy of less than $\sim 200 \mathrm{Myr}$. The brightest emission lines are clearly asymmetric and are well fit by two Gaussian components separated by $\sim 22 \mathrm{~km} \mathrm{~s}^{-1}$. We detect two discrete Na I and Ca II absorption components at the same redshifts as the emission components. We tentatively interpret the two components as arising from two different starforming regions in the host, but high resolution imaging is necessary to confirm this.
\end{abstract}

Key words. gamma rays: bursts - galaxies: high redshift - galaxies: abundances - cosmology: observations

\section{Introduction}

Long-duration gamma-ray bursts (GRBs) are widely accepted to be related to core-collapse supernovae: clear supernova signatures are seen in the afterglow spectra of low redshift GRBs (e.g. Stanek et al. 2003; Hjorth et al. 2003; Pian et al. 2006). Dedicated surveys of GRB hosts suggest that GRBs occur preferentially in low mass, subluminous, blue star-forming galaxies (e.g. Chary et al. 2002; Fruchter et al. 1999; Le Floc'h et al. 2003). The GRBs are often located within UV-bright parts of their hosts, where star formation takes place (Bloom et al. 2002; Fruchter et al. 2006), and are shown to be more concentrated towards the brightest regions of their hosts than are, in general, core-collapse supernovae (Fruchter et al. 2006).

GRB host galaxies are not selected through their luminosities or colours, but merely by the fact that a GRB has been detected. This could potentially provide an unbiased sample of

^ Based on observations obtained at the ESO VLT under ESO programme 076.A-0737(A). starforming galaxies, which may be used to study star formation in the Universe (see e.g. Jakobsson et al. 2005). The significant increase in detection rate and localisation of long GRBs through the successful operation of Swift in principle permits the study of a large and uniformly selected sample of GRB host galaxies. However, to date the sample of spectroscopically studied GRB host galaxies is small ( $\sim 30$ cases) and may suffer from several selection biases, due to their faintness.

One of the key properties needed to understand GRB progenitors and their environments is the metallicity distribution (e.g. Langer \& Norman 2006; Yoon et al. 2006): the popular collapsar model for long GRBs requires low metallicity progenitors. Metallicities for GRB hosts can be determined through afterglow spectroscopy and through host galaxy spectroscopy. Absorption lines of H I and heavy metals have provided metallicities along GRB sight-lines in the redshift interval $2<z<6.2$.

Host galaxy spectroscopy can provide metallicities for the galaxy as a whole, but relies on the detection of the nebular emission lines, which is difficult at $z \gtrsim 1$. There are only a few 
GRB host galaxies that are bright enough to permit a direct abundance analysis through an electron temperature $\left(T_{\mathrm{e}}\right)$ determination (GRBs 980425, 020903, 031203; Prochaska et al. 2004; Hammer et al. 2006). It is only possible to study the metallicities of higher redshift and fainter hosts through secondary metallicity indicators using bright (nebular) lines (e.g. the R23 method), using empirical correlations between the fluxes of certain emission lines and metallicity.

On the 18th of February 2006 a bright, nearby GRB was discovered by Swift. The proximity $(z \sim 0.0334$, Mirabal \& Halpern 2006, the second closest GRB) of this GRB triggered a large follow-up campaign by several groups, which provided a unique opportunity to unveil the nature of a faint nearby galaxy associated with a GRB event. GRB 060218 was found to be unusually long in duration $\left(T_{90}=2100 \pm 100 \mathrm{~s}\right.$, Campana et al. 2006) and of relatively low luminosity $\left(E_{\gamma, \text { iso }}=6.2 \pm 0.3 \times 10^{49} \mathrm{erg}\right.$, Soderberg et al. 2006). Its prompt emission was soft, placing it in the class of X-ray flashes (XRFs). A bright supernova (designated SN 2006aj) was clearly associated with this event which was studied with very high spectral and time resolution over a wide range of wavelengths, from X-rays to radio (e.g. Campana et al. 2006; Pian et al. 2006; Mazzali et al. 2006; Soderberg et al. 2006). The host was found to be a small galaxy with an irregular morphology.

In this paper we study the host of GRB 060218 through a high resolution VLT Ultraviolet and Visual Echelle Spectrograph (UVES; Dekker et al. 2000) spectrum, taken around the peak magnitude of the supernova. This spectrum shows a variety of well resolved emission lines, associated with ionized gas in the starforming regions in the host. The neutral gas of the ISM is probed by the detection of a few narrow absorption lines. Low resolution FORS spectra (described in Pian et al. 2006) are used to study the fluxes of emission lines that fall outside the spectral coverage of the UVES spectrum.

Several papers have already been published on GRB 060218, its associated supernova, and the host. However, on the metallicity of the host there is a wide range of reported values. Modjaz et al. (2006) report a metallicity of $0.15 Z_{\odot}$, Mirabal et al. (2006) derive $0.46 Z_{\odot}$, while Sollerman et al. (2006) mention that the abundance is below Solar, but that the exact value is unconstrained from the strong emission lines. These values are derived from different spectra, but the spread is largely due to internal scatter in empirical, secondary calibrators that are used, and the limited range of metallicities and diagnostic emission line ratios for which these secondary calibrators are valid (see e.g. Ellison $\&$ Kewley 2006). Since the derived metallicities are frequently used to draw conclusions on important issues such as progenitors (e.g. Sollerman et al. 2005) or the rate of nearby GRBs (Stanek et al. 2006; Wolf \& Podsiadlowski 2006), it is clearly of great interest to pin down these uncertainties.

The paper is organized as follows: in Sect. 2 we describe the observations. In Sect. 3 we calculate the metallicity of the dominant starforming region(s) in the host galaxy and relative abundances of $\mathrm{Ne}$ and $\mathrm{N}$, as well as the star formation rate from optical and radio fluxes. In Sect. 4 we analyse the discrete velocity components in the host through emission and absorption lines. We discuss the use of metallicity values found from secondary metallicity calibrators in Sect. 5. In Sect. 6 we discuss the implications that a future detection of Wolf-Rayet star signatures in the host of GRB 060218 may have on single star GRB progenitor models.

Throughout this paper we use the cosmological parameters $H_{0}=70 \mathrm{~km} \mathrm{~s}^{-1} \mathrm{Mpc}^{-1}, \Omega_{\mathrm{M}}=0.3$ and $\Omega_{\Lambda}=0.7$.

\section{Observations}

GRB 060218/SN 2006aj was observed with ESO VLT Kueyen (UT 2) on March 4, 2006, roughly at the time of maximum light of the supernova (around March 1, e.g. Sollerman et al. 2006). The UVES observation started at 00:30 UT, for a total exposure time of $2100 \mathrm{~s}$. The magnitude of SN 2006aj was $V \sim 17.6$ at the time of observation. The airmass was high, averaging 2.5, and the seeing measured from the 2D spectrum is 1.1 arcsec. The UVES-setup spectral resolution is $R \sim 46000$ (FWHM $\simeq$ $6.5 \mathrm{~km} \mathrm{~s}^{-1}$ ). The slit width was set at 1 arcsec, corresponding to about $7 \mathrm{kpc}$ physical size at the distance of the GRB. The exposure was performed at the parallactic angle. The spectrum (wavelength ranges 3285-4527 $\AA, 4621-5598 \AA$ and 5676-6651 $)$ was reduced in the standard fashion using MIDAS and IRAF routines.

The L.A.Cosmic program (specifically the lacos_spec routine, Van Dokkum 2001) was used to remove both point- and irregular shaped cosmic ray hits from the $2 \mathrm{D}$ spectrum before extraction. The spectrum was dispersion corrected, and flux calibrated by archived response functions. An air to vacuum conversion and a heliocentric correction $\left(+28 \mathrm{~km} \mathrm{~s}^{-1}\right)$ were applied to the spectrum. The resulting spectrum was compared with a (quasi)-simultaneous FORS spectrum taken 20 min after the UVES spectrum, which is flux calibrated through a standard star observation and through simultaneous photometry in $B, V, R$ at VLT FORS2 (Pian et al. 2006), using the magnitude to flux conversions from Fukugita et al. (1995). To the UVES spectrum a multiplication factor of 1.625 was applied to match the well calibrated FORS flux values (compensating for the slit loss between the FORS and UVES spectra).

We find a good match between the UVES and FORS spectra and photometry in the red end (above $\lambda \sim 4500 \AA$ ), as shown in Fig. 1. In the blue end $(\lambda \lesssim 4500 \AA)$ the UVES continuum flux is slightly higher than the FORS continuum flux. The prime reason is likely the very high airmass at which both spectra have been taken, making the flux calibration of both the UVES and FORS spectrum at the blue end more uncertain. We decide to not alter the flux calibration, but warn that the fluxes of emission lines below $\sim 4500 \AA$ have a small additional uncertainty. This uncertainty does not significantly affect the metallicity results of this paper, because the electron temperature uncertainty is dominated by the uncertainty in the flux of [O III] $\lambda 4364$ from the FORS spectra. The host galaxy emission line flux ratios found in the UVES spectrum agree, within the errors, with those found from the combination of the FORS spectra.

A Galactic extinction correction was performed using $E(B-$ $V)_{\mathrm{MW}}=0.142$ (Schlegel et al. 1998), assuming a Galactic extinction law $A_{\lambda} / A_{V}$ expressed as $R_{V}=A_{V} / E(B-V)$ (Cardelli et al. 1989), and $R_{V}=3.1$. This value is slightly higher than the extinction derived by Guenther et al. (2006), who used the Galactic sodium lines to find $E(B-V)_{\mathrm{MW}}=0.127$. Given that the systematic error in the conversion of the equivalent widths of $\mathrm{Na}$ to $E(B-V)$ is poorly known, we choose to use $E(B-V)_{\mathrm{MW}}=0.142 \mathrm{mag}$.

A selection of lines from the spectrum and the associated error spectra are shown in Figs. 2 and 5. Although the spectrum is dominated by the SN spectrum $(V \approx 17.6$ vs. the host $V \approx 20.2$ ), several bright emission lines from the host galaxy are detected and resolved in the UVES spectrum (Fig. 2, Table 3). On the other hand, the detected absorption lines, from both the host galaxy and Milky Way, are narrower than the UVES resolving power. The highest signal-to-noise emission lines provide a heliocentric mean redshift $z=0.03342$ (2) (Pian et al. 2006), 


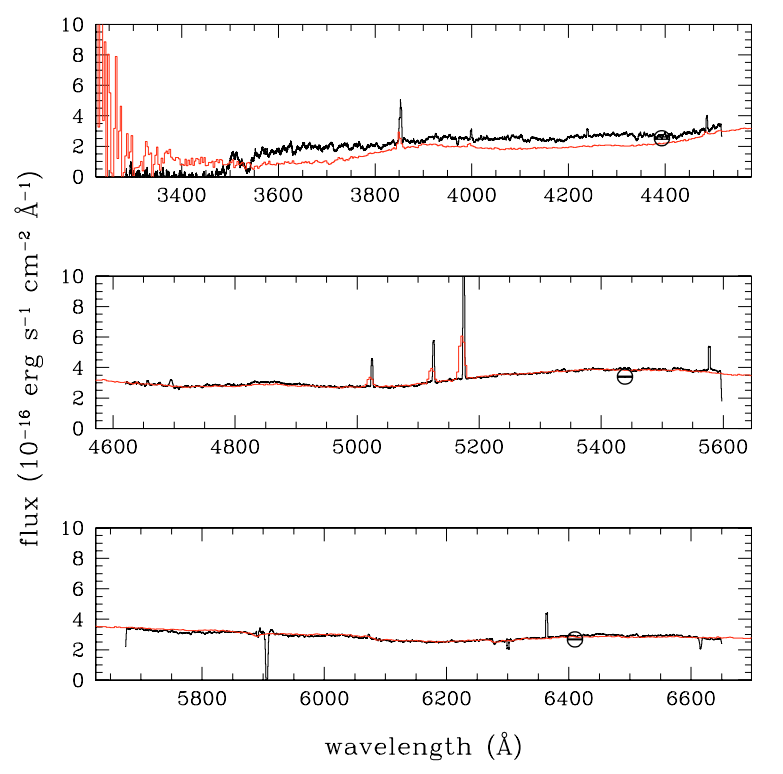

Fig. 1. The UVES spectrum shown in black, with overlaid in red the low resolution FORS spectrum. For presentation purposes the UVES spectrum has been smoothed to the pixelscale of the FORS spectrum. The points denote $B, V, R$ VLT FORS photometry from the same night (Pian et al. 2006). The widths of the broadband filters are not shown.

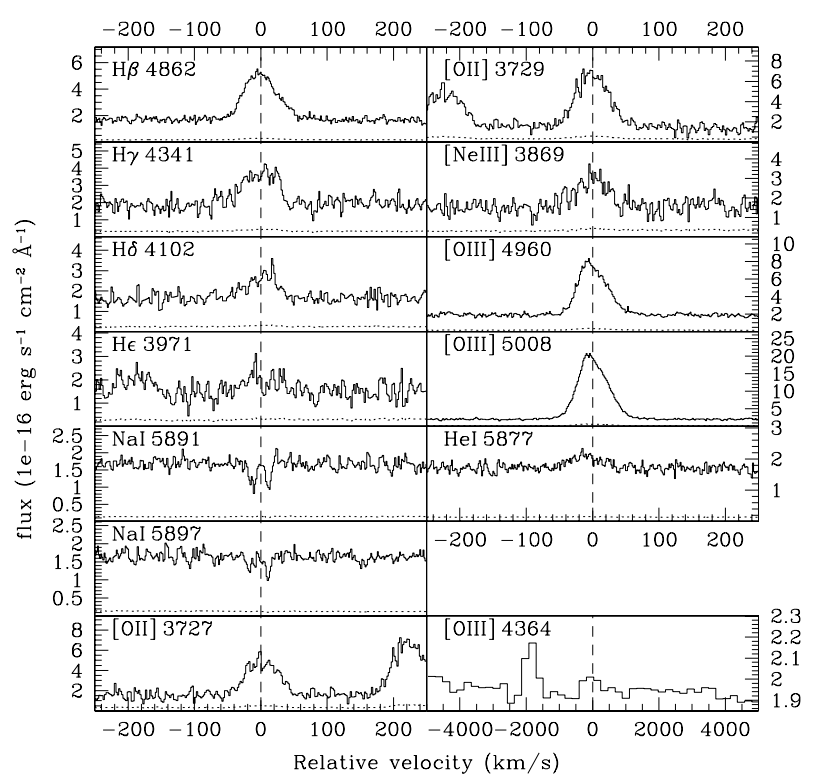

Fig. 2. Emission and absorption lines detected in the UVES spectrum that were used to derive the host properties. The error spectra are plotted with dotted lines. The vertical dashed lines denote the mean redshift of the host. The bottom right panel shows the [O III] $\lambda 4364$ line from the FORS spectrum.

where we adopt a conservative error on the redshift due to poorly known systematic effects of the spectrograph (e.g. the centering of the object in the slit).

The analysis of the emission lines was done using the IRAF software packages, mainly using the splot routines. As the emission lines are clearly asymmetric (see Sect. 4.1), fluxes are measured using the numerical integration method ( $e$ in splot), and not the standard Gaussian fitting. The errors in the line fluxes are generally dominated by the uncertainty in the continuum level and are given at the $1 \sigma$ level.

\section{General host galaxy properties}

\subsection{Metallicity}

\subsubsection{Reddening}

Before we can use the emission lines in the FORS and UVES spectra to derive the host properties, we need to correct for the dust extinction intrinsic to the host. We use the Balmer line fluxes to measure a value for the Balmer decrement, assuming case B recombination (e.g. Osterbrock 1989; Izotov et al. 1994). The detection of several of the Balmer lines in the UVES spectrum, see Table 3, provides a good constraint on the extinction value. We assume intrinsic Balmer line ratios at $20000 \mathrm{~K}$, and compute flux ratios of the detected Balmer lines. We caution that $\mathrm{H} \delta$ and $\mathrm{H} \epsilon$ are located in the blue end of the UVES spectrum, where we see a slight discrepancy in flux calibration between the FORS spectrum of March 4 and the UVES spectrum (the UVES spectrum having a slightly higher mean continuum flux). We find that the Balmer line ratios from the UVES spectrum are all $(\mathrm{H} \beta / \mathrm{H} \gamma$ to $\mathrm{H} \beta / \mathrm{H} \epsilon)$ consistent with the theoretical case B recombination values. The FORS spectra are taken at low resolution $(R \sim 300)$, making the extinction derivation based on the $\mathrm{H} \alpha / \mathrm{H} \beta$ ratio from these data less reliable. We test various intrinsic stellar Balmer absorption strengths, but find no evidence for internal reddening from the Balmer decrement in the UVES spectrum, with upper limit $E(B-V) \lesssim 0.03$. However this value may be influenced by the uncertain flux calibration of the blue end of the spectrum, see Sect. 2. The $\mathrm{H} \beta$ and $\mathrm{H} \gamma$ lines are located in an area of the spectrum where the FORS and UVES continuum fluxes are more in agreement. The ratio of the $\mathrm{H} \beta$ and $\mathrm{H} \gamma$ lines is consistent with case $\mathrm{B}$ recombination values, $E(B-V) \lesssim 0.04$ (1 sigma). In Guenther et al. (2006) a reddening of $E(B-V)=0.042 \pm 0.003$ is derived from the strengths of the sodium absorption lines in the host, and used by Pian et al. (2006) to account for reddening in the host. We choose to use the more common method of accounting for extinction by using the Balmer decrement. The resulting net extinction (Galactic + host) is close to the value applied by Pian et al. (see also Sollerman et al. 2006, for a discussion) and does not affect further analysis, as the uncertainties in the line ratios are dominated by the uncertainties in their fluxes. We further note that the high electron temperature seen in this source (see Sect. 3.1.2) can make collisional excitation of neutral hydrogen important, which can mimic reddening and affect the Balmer decrement derived reddening. We can not reliably evaluate this effect, as we do not measure a significant Balmer decrement.

We use various nebular line flux ratios to evaluate the possibility of an Active Galactic Nucleus (AGN) contribution to the excitation of the nebular lines. Kauffmann et al. (2003) refine the popular line ratio diagnostic [O III] $\lambda 5008 / \mathrm{H} \beta$ vs. [N II] $/ \mathrm{H} \alpha$, by analyzing a large sample of galaxies from the SDSS. They empirically define the demarcation between starburst galaxies and AGN as follows: a galaxy is AGN dominated if

$\log ([\mathrm{O}$ III $] \lambda 5008 / \mathrm{H} \beta)>0.61 /(\log ([\mathrm{N} \mathrm{II}] / \mathrm{H} \alpha)-0.05)+1.3$.

Kauffmann et al. (2003) use extinction corrected fluxes, but note that these line ratios are relatively insensitive to extinction effects. We use the flux values measured from the FORS spectra, [O III] $\lambda 5008 / \mathrm{H} \beta=4.55 \pm 0.28$ and $[\mathrm{N} \mathrm{II}] / \mathrm{H} \alpha=0.06 \pm 0.01$. These flux ratios place the host galaxy comfortably in the locus of actively starforming galaxies. Hence we assume that the host galaxy nebular emission line excitation is not dominated by (non-thermal) AGN emission. 


\subsubsection{Electron density and temperature}

We make the standard two zone model assumption for the H II region(s) from which the emission lines originate, consisting of a low and intermediate temperature region (e.g. Osterbrock 1974). The [O II] $\lambda \lambda 3726$ and $3729 \AA$ lines (Fig. 2) are closely linked to collisional excitation and de-excitation at the typical temperatures of star forming regions $\left(\sim 10^{4} \mathrm{~K}\right)$. The electron density $\left(n_{\mathrm{e}}\right)$ in the low temperature region can be derived from the ratio of the fluxes of these two lines (Osterbrock 1974). The ratio of the line fluxes $\lambda 3726 / \lambda 3729$ approaches $\frac{4}{6}$ in the $n_{\mathrm{e}} \rightarrow 0$ limit. In the limit $n_{\mathrm{e}} \rightarrow \infty$ the flux ratio approaches 2.86 (collisional excitation and de-excitation dominate over radiative transitions, forming a Boltzmann population ratio). As shown in Fig. 2, in the UVES spectrum the [O II] doublet is clearly resolved, and its flux ratio is $0.62 \pm 0.05$. This number is consistent with the low density limit, implying that collisional de-excitation is not important for the fluxes of the forbidden lines. The resolved [O II] doublet has also been observed in two other GRB host galaxy spectra, GRBs 990506 and 000418, which have values of [O II] $\lambda 3726 / \lambda 3729$ of $0.57 \pm 0.14$ and $0.75 \pm 0.11$, respectively (Bloom et al. 2002). These two detections imply low values for $n_{\mathrm{e}}$ similar to GRB 060218 .

A different doublet used frequently as a density diagnostic is the [S II] $\lambda \lambda 6717,6731$ doublet. The line ratio [S II] $\lambda 6717 / \lambda 6731$ approaches 1.5 when $n_{\mathrm{e}} \rightarrow 0$, and 0.44 above $n_{\mathrm{e}} \sim 10^{4} \mathrm{~cm}^{-3}$. These lines are redshifted out of the UVES wavelength range, but are detected in the FORS spectra. We find $[\mathrm{S} \mathrm{II}] \lambda 6717 / \lambda 6731=1.0 \pm 0.6$, which is therefore not useful to discriminate between the high and low density regimes. Prochaska et al. (2004) find a ratio of $1.19 \pm 0.09$ for the host of GRB 031203, corresponding to $n_{\mathrm{e}} \sim 300 \mathrm{~cm}^{-3}$. We assume the relatively low values of $n_{\mathrm{e}}=100 \mathrm{~cm}^{-3}$ for our analysis, following e.g. Skillman et al. (1994); Izotov et al. (2006b).

We estimate the electron temperature $\left(T_{\mathrm{e}}\right)$ in the intermediate temperature region from the ratio of [O III] nebular and auroral fluxes. The auroral [O III] $\lambda 4364$ is not significantly detected in the UVES spectrum - it is located in a noisy region close to the gap in between the wavelength ranges, see Sect. 2 .

From the FORS spectrum we find the flux ratio [O III] $(\lambda 4959+\lambda 5008) / \lambda 4364=29.1 \pm 6.3$. The relatively large error is mainly due to the rather large uncertainty in the [O III] $\lambda 4364$ flux value. We use the electron density assumed above, which we take to be the same in both the low- and intermediate temperature regions (see e.g. Osterbrock 1974), and find an electron temperature $T_{\mathrm{e}}\left(\mathrm{O}^{2+}\right)$ in the intermediate temperature region of $2.48_{-0.3}^{+0.5} \times 10^{4} \mathrm{~K}$. This is high when compared to that of the host of GRB $031203\left(T_{\mathrm{e}}\left(\mathrm{O}^{2+}\right) \sim 13400 \mathrm{~K}\right.$, Prochaska et al. 2004), as shown in Table 1. Comparably high values of $T_{\mathrm{e}}\left(\mathrm{O}^{2+}\right)$ have been observed in the recent discovery of two extremely low metallicity galaxies by Izotov et al. (2006b). This very high temperature and low density suggests a low oxygen abundance for the host of GRB 060218, since the main nebular cooling is done through oxygen forbidden line emission.

Especially at low metallicity there can be large differences in electron temperature between the low and high temperature zones. Due to a lack of detected lines that can be used as temperature indicators in the low temperature region (the [O II] $\lambda \lambda 7320,7331$ are redshifted out of the UVES coverage and are too faint for the FORS spectrum), we follow the recipe by Izotov et al. (2006a),

$T_{\mathrm{e}}\left(\mathrm{O}^{+}\right)=-0.577+T_{\mathrm{e}}\left(\mathrm{O}^{2+}\right) \times\left(2.065-0.498 T_{\mathrm{e}}\left(\mathrm{O}^{2+}\right)\right)$, where $T_{\mathrm{e}}$ is in units of $10^{4} \mathrm{~K}$. We find $T_{\mathrm{e}}\left(\mathrm{O}^{+}\right)=1.5_{-0.2}^{+0.1} \times$ $10^{4} \mathrm{~K}$, using the approximation for a low metallicity environment $(12+\log \mathrm{O} / \mathrm{H} \sim 7.2$, see Izotov et al. 2006a). When we assume an intermediate metallicity $(12+\log \mathrm{O} / \mathrm{H} \sim 7.6)$, we find $T_{\mathrm{e}}\left(\mathrm{O}^{+}\right)=1.3_{-0.5}^{+0.2} \times 10^{4} \mathrm{~K}$. We adopt the low metallicity value for now, and note that the prescription by Pagel et al. (1992) gives a similar value of $T_{\mathrm{e}}\left(\mathrm{O}^{+}\right)=1.66_{-0.08}^{+0.1} \times 10^{4} \mathrm{~K}$. The conversion above from $T_{\mathrm{e}}\left(\mathrm{O}^{2+}\right)$ to $T_{\mathrm{e}}\left(\mathrm{O}^{+}\right)$is derived through sequences of photoionization models (Stasińska \& Izotov 2003). Izotov et al. (2006a) show by comparing this conversion with direct measurements of $T_{\mathrm{e}}\left(\mathrm{O}^{2+}\right)$ and $T_{\mathrm{e}}\left(\mathrm{O}^{+}\right)$that the models agree with the measurements. There is a significant scatter of direct measurements of $T_{\mathrm{e}}\left(\mathrm{O}^{+}\right)$and $T_{\mathrm{e}}\left(\mathrm{O}^{2+}\right)$ with respect to the model predictions, which is probably mainly due to the large uncertainties in the measurements of $T_{\mathrm{e}}\left(\mathrm{O}^{+}\right)$(Izotov et al. 2006a). We do not include an additional uncertainty in the following analysis to account for this, but note that the small errors reported here should not be overinterpreted. We assume the $T_{\mathrm{e}}\left(\mathrm{O}^{+}\right)$to also be valid for $\mathrm{N}$ II and S II, which have comparable ionisation potentials.

\subsubsection{Oxygen abundance}

We use the equations from Izotov et al. (2006a) to derive ionic abundances from the derived electron densities and temperatures. To find the oxygen abundance, $\mathrm{O} / \mathrm{H}$, we sum the $\mathrm{O}^{2+} / \mathrm{H}^{+}$and $\mathrm{O}^{+} / \mathrm{H}^{+}$ion abundances, assuming the $\mathrm{O}^{3+}$ abundance is negligible since no high temperature lines (e.g. He II) were detected. We take the line fluxes from the UVES spectrum, and find $\mathrm{O}^{2+} / \mathrm{H}^{+}=1.72 \pm 0.45 \times 10^{-5}$ and $\mathrm{O}^{+} / \mathrm{H}^{+}=$ $1.78_{-0.32}^{+1.2} \times 10^{-5}$. The errors include the uncertainties on the line flux ratios and electron temperature. The ratio $\mathrm{O}^{+} /\left(\mathrm{O}^{+}+\right.$ $\left.\mathrm{O}^{2+}\right)>0.1$, confirming that the excitation is too low for $\mathrm{O}^{3+}$ to be important, as shown by photoionization models by Izotov et al. (2006). The large uncertainty in the $\mathrm{O}^{+} / \mathrm{H}^{+}$abundance is caused by the relatively large error in the $T_{\mathrm{e}}\left(\mathrm{O}^{+}\right)$. The total oxygen abundance is $\mathrm{O} / \mathrm{H}=\mathrm{O}^{2+} / \mathrm{H}^{+}+\mathrm{O}^{+} / \mathrm{H}^{+}=3.50$ ${ }_{-0.77}^{+1.65} \times 10^{-5}$, or $12+\log (\mathrm{O} / \mathrm{H})=7.54_{-0.1}^{+0.16}$, or $\simeq 0.07 \mathrm{Z}_{\odot}$, assuming $\log (\mathrm{O} / \mathrm{H})_{\odot}+12=8.69$ (Allende Prieto et al. 2001). This value places the host of GRB 060218 among the most metal deficient subset of emission line galaxies in the local universe (e.g. Izotov et al. 2006a; Lee et al. 2006b). It is also the lowest metallicity found so far for a GRB host from emission line analysis (absorption line metallicities from several afterglows show lower line of sight metallicities, down to $\sim 0.01 Z_{\odot}$ for GRB 050730, Starling et al. 2005; Chen et al. 2005). We note that in an independent analysis Kewley et al. (2006) found a similar value for the metallicity of the host of GRB 060218 of $12+\log (\mathrm{O} / \mathrm{H}) \sim 7.6$.

\subsection{Relative element abundances}

Accurate emission-line abundances have been derived for a small sample of GRB host galaxies. A notable example is the spectrum of the host of GRB 031203, for which a solar abundance pattern was established (Prochaska et al. 2004). We use the host emission lines measured in the UVES and FORS spectra to gain an insight into the abundance pattern in the host of GRB 060218.

The detection of the forbidden [Ne III] lines allows us to derive a Ne abundance, using the values for $n_{\mathrm{e}}$ and $T_{\mathrm{e}}\left(\mathrm{O}^{2+}\right)$ found in Sect. 3.1. We use the [Ne III] $\lambda 3869 / \mathrm{H} \beta$ flux ratio from the UVES spectrum and find $\mathrm{Ne}^{2+} / \mathrm{H}^{+}=2.9 \pm 1.2 \times 10^{-6}$, 
Table 1. Table of properties of the hosts of GRB 060218 (this work) and GRB 031203 (Prochaska et al. 2004) and a comparison to the northwest and southeast regions of I Zw 18 (Izotov et al. 1999).

\begin{tabular}{|c|c|c|c|c|}
\hline Property & GRB 060218 & GRB 031203 & I Zw $18 \mathrm{NW}$ & I Zw18 SE \\
\hline$T_{\mathrm{e}}(\mathrm{O}$ III $)$ & $2.48_{-0.3}^{+0.5} \times 10^{4} \mathrm{~K}$ & $13400 \pm 2000$ & $19780 \pm 640$ & $19060 \pm 610$ \\
\hline$T_{\mathrm{e}}(\mathrm{O}$ II $)$ & $1.5_{-0.2}^{+0.1} \times 10^{4} \mathrm{~K}$ & 12900 & $15620 \pm 470$ & $15400 \pm 460$ \\
\hline $\mathrm{O}^{2+} / \mathrm{H}^{+}$ & $1.72 \pm 0.45 \times 10^{-5}$ & - & $1.216 \pm 0.09 \times 10^{-5}$ & $1.106 \pm 0.082 \times 10^{-5}$ \\
\hline $\mathrm{O}^{+} / \mathrm{H}^{+}$ & $1.78_{-032}^{+1.2} \times 10^{-5}$ & - & $0.179 \pm 0.014 \times 10^{-5}$ & $0.403 \pm 0.031 \times 10^{-5}$ \\
\hline $\mathrm{O} / \mathrm{H}$ & $3.50_{-0.77}^{+1.65} \times 10^{-5}$ & - & $1.465 \pm 0.092 \times 10^{-5}$ & $1.523 \pm 0.088 \times 10^{-5}$ \\
\hline $12+\log (\mathrm{O} / \mathrm{H})$ & $7.54_{-01}^{+0.16}$ & $8.02 \pm 0.15$ & $7.166 \pm 0.027$ & $7.183 \pm 0.025$ \\
\hline $\mathrm{N}^{+} / \mathrm{H}^{+}$ & $1.4_{-0.4}^{+0.8} \times 10^{-6}$ & - & - & $1.074 \pm 0.084 \times 10^{-7}$ \\
\hline $\operatorname{ICF}(\mathrm{N})$ & 1.98 & - & - & 3.78 \\
\hline $\log (\mathrm{N} / \mathrm{O})$ & $-1.1 \pm 0.4$ & $-0.74 \pm 0.2$ & - & $-1.574 \pm 0.06$ \\
\hline $\mathrm{Ne}^{2+} / \mathrm{H}^{+}$ & $2.9 \pm 1.2 \times 10^{-6}$ & - & $1.91 \pm 0.16 \times 10^{-6}$ & $1.83 \pm 0.15 \times 10^{-6}$ \\
\hline $\mathrm{ICF}(\mathrm{Ne})$ & 1.11 & - & 1.20 & 1.28 \\
\hline $\log (\mathrm{Ne} / \mathrm{O})$ & $-0.8_{-0.4}^{+0.2}$ & $-0.85 \pm 0.2$ & $-0.803 \pm 0.053$ & $-0.781 \pm 0.051$ \\
\hline
\end{tabular}

and $\mathrm{Ne}^{2+} / \mathrm{O}^{2+}=0.17 \pm 0.11$, which is consistent with other low metallicity H II regions, e.g. in I Zw 18 (where $\mathrm{Ne}^{2+} / \mathrm{O}^{2+} \sim$ 0.13 , see Table 1$)$. To derive the $\mathrm{Ne} / \mathrm{H}$ abundance we need an ionisation correction function (ICF) for which we use the parametrization by Izotov et al. (2006a). We find ICF $\left(\mathrm{Ne}^{2+}\right)=1.11$ and $\mathrm{Ne} / \mathrm{H}=3.3 \pm 1.3 \times 10^{-6}$.

The [S III] $\lambda 6312$ line is not detected in the UVES or FORS spectra and [S III] $\lambda \lambda 9532,9069$ are redshifted out of both the UVES and FORS coverage. We will therefore only derive a value for the ionic $\mathrm{S}^{+}$abundance, and give an upper limit for the total sulphur abundance. We use $T_{\mathrm{e}}\left(\mathrm{O}^{+}\right)$and find $\mathrm{S}^{+} / \mathrm{H}^{+}=$ $2.7_{-1.0}^{+2.0} \times 10^{-7}$. For the limit on $\mathrm{S}^{2+} / \mathrm{H}^{+}$we transform $T_{\mathrm{e}}\left(\mathrm{O}^{2+}\right)$ to $T_{\mathrm{e}}\left(\mathrm{S}^{2+}\right)$ through the recipe of Izotov et al. (2006a), and use the upper limit on the [S III] flux from the UVES spectrum to find $\mathrm{S}^{2+} / \mathrm{H}^{+}<2.2 \times 10^{-6}$. We calculate an $\operatorname{ICF}\left(\mathrm{S}^{+}+\mathrm{S}^{2+}\right)$ of $\sim 1$ which allows us to set the not particularly constraining limit $\log (\mathrm{S} / \mathrm{O})<-1.1$ (Solar value is $\log (\mathrm{S} / \mathrm{O})=-1.50$, Lodders 2003), which is consistent with the observed trend for $\mathrm{S}$ to follow Solar $(\mathrm{S} / \mathrm{O})$ ratios independent of $(\mathrm{O} / \mathrm{H})$ for low metallicity $\mathrm{H}$ II regions.

The [N II] $\lambda 6584$ line is redshifted out of the UVES range, but is detected in the FORS spectrum. The weaker [N II] $\lambda 6548$ line is not significantly detected in the FORS spectrum, with a $3 \sigma$ upper limit on the flux of $\sim 8 \times 10^{-17} \mathrm{erg} \mathrm{s}^{-1} \mathrm{~cm}^{-2}$. We use the fixed flux ratio $\lambda 6584 / \lambda 6548=2.9$ (Osterbrock 1989) and $T_{\mathrm{e}}\left(\mathrm{O}^{+}\right)$, and find $\mathrm{N}^{+} / \mathrm{H}^{+}=1.4_{-0.4}^{+0.8} \times 10^{-6}$.

To calculate $\mathrm{N} / \mathrm{H}$ from $\mathrm{N}^{+} / \mathrm{H}^{+}$we correct for ionisation using ICF $\left(\mathrm{N}^{+}\right)=1.98$, and find $\mathrm{N} / \mathrm{H}=2.8_{-0.8}^{+1.6} \times 10^{-6}$, see Fig. 3 . The ratio $\mathrm{N}^{+} / \mathrm{O}^{+}=0.08_{-0.05}^{+0.07}$ is comparable to, though slightly above, the ratio for $\mathrm{I} Z \mathrm{~W} 18$ of $\mathrm{N}^{+} / \mathrm{O}^{+} \sim 0.03$. We note that here we compare the abundances of two elements using two different spectra (UVES and FORS) taken under different conditions, and the uncertainty on $\log (\mathrm{N} / \mathrm{O})$ is likely underestimated. Nevertheless the N/O ratio does not significantly deviate from the observed trend of low metallicity galaxies to have $\log (\mathrm{N} / \mathrm{O}) \sim-1.5$ (e.g. Lopez \& Ellison 2003; Izotov et al. 2006).

Hammer et al. (2006) observed the host of GRB 980425/SN 1998bw with significant spatial resolution (owing to the large spatial extent of this host galaxy), and found a high ratio $\log (\mathrm{N} / \mathrm{O})=-0.6$ from a $T_{\mathrm{e}}$ abundance analysis at the region at which the GRB / SN took place, which corresponds to almost twice the Solar value. This is unexpected at the measured metallicity, see Fig. 3. Prochaska et al. (2004) find a similarly high value of $\log (\mathrm{N} / \mathrm{O})=-0.74 \pm 0.2 \mathrm{dex}$ in their spectrum of the host of GRB 031203, at a metallicity of

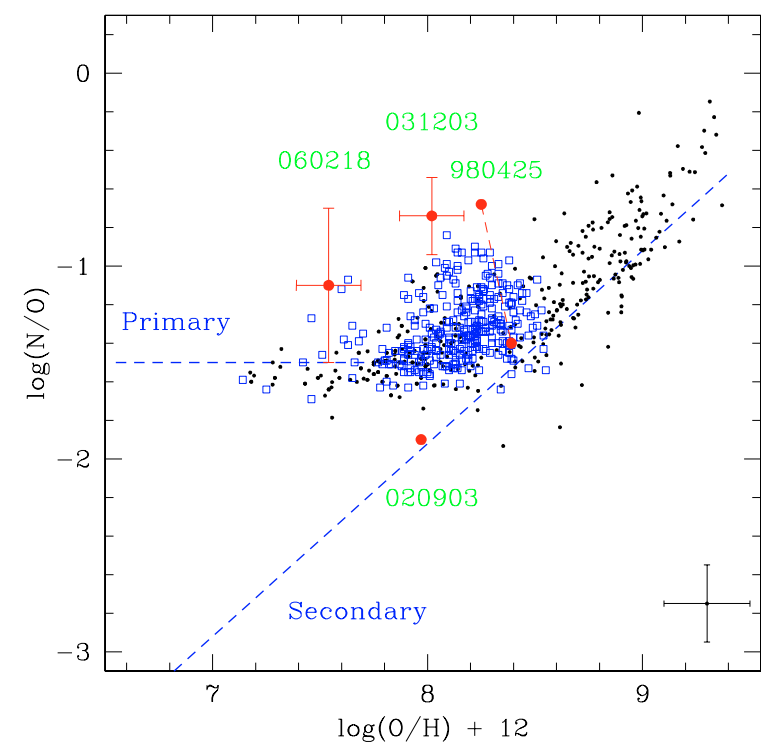

Fig. 3. Measurements of $\log (\mathrm{N} / \mathrm{O})$ and $12+\log (\mathrm{O} / \mathrm{H})$ from a sample of galaxies from the SDSS-DR3 (Izotov et al. 2006) are shown with open squares, together with points from Lopez \& Ellison (2003) (small points), for which typical uncertainties are indicated in the bottom right corner. The blue dashed lines show the tracks where primary and secondary nitrogen dominate the N/O ratio. Published GRB host galaxy measurements are overplotted: GRB 060218 (this work), GRB 031203 (Prochaska et al. 2004), GRB 980425 (the dashed line connects the value for the $\mathrm{SN}$ region with the higher $\log (\mathrm{N} / \mathrm{O})$ and the general host value, see Sect. 3.2, Hammer et al. 2006) and GRB 020903 (Hammer et al. 2006).

$\log (\mathrm{O} / \mathrm{H})+12=8.1$. GRB 020903 shows a value more in line with expectation from its metallicity (see Fig. 3). In the case of GRB 060218 the uncertainty on the $(\mathrm{N} / \mathrm{O})$ ratio is too high to exclude a deviation from the expected value at the metallicity of the host. A possible high N/O value can be explained by a variety of reasons. If $T_{\mathrm{e}}$ has been highly overestimated the metallicity would decrease, moving the points in Fig. 3 to the left. Physical reasons for an enhanced N/O ratio may be e.g. a contribution of shock heating to the line emission or a chemical evolution effect: Hammer et al. (2006) explain the higher N/O ratio at the locus of GRB 980425 by a larger $\mathrm{N}$ yield of a GRB progenitor or $\mathrm{SN}$ remnants.

The hosts of GRBs 980425, 031203, 020903 and 060218 form a sequence in metallicity, from the metallicities where we expect both primary and secondary nitrogen production to play 
an active role (the host of GRB 980425) to where primary $\mathrm{N}$ is expected to dominate (as in the host of GRB 060218). This makes the GRB 060218 host an interesting candidate for deep spectroscopy to obtain a more accurate N/O when the SN has fully faded.

The measured nitrogen, oxygen and neon abundances derived for the host galaxy of GRB 060218 are shown in Table 1. We note that these are not spatially resolved. Izotov et al. (1999) noted that in the case of I Zw 18 a gradient in electron temperature can be seen, with the highest temperatures in the regions where WR stars are found. These differences in temperature are associated with significant differences in (oxygen) abundance (with factors up to $\sim 1.4$, Izotov et al. 1999). This gradient may be due to oxygen enrichment by starforming clusters, and incomplete mixing in the galaxy. Without spatial information we can not check abundance gradients in most GRB hosts, and assume the oxygen abundances found are representative for the galaxy as a whole, including the progenitor locus. However, Hammer et al. (2006) and Sollerman et al. (2006) have shown that in the case of the host of SN 1998bw strong differences in $T_{\mathrm{e}}$ and abundances are observed as well.

\subsection{Star formation}

The detection of the bright $\mathrm{H} \alpha \lambda 6563$ emission line in the FORS spectrum allows us to accurately measure star formation in the host: the $\mathrm{H} \alpha$ line luminosity only weakly depends on the physical conditions of the ionized gas. We use

$S F R_{\mathrm{H} \alpha}=7.9 \times 10^{-42} L_{\mathrm{H} \alpha}$,

as found by Kennicutt (1998). Moustakas et al. (2006) assess the accuracy of this expression through a direct comparison of extinction corrected $L_{\mathrm{H} x}$ SFR values and the SFR derived from $L_{\mathrm{IR}}$ measurements of a sample of IRAS detected galaxies. They find that when the $\mathrm{H} \alpha$ flux is extinction corrected, the IR and $\mathrm{H} \alpha$ SFRs agree without systematic offset with a precision of $\sim 70 \%$. The extinction corrected flux of $\mathrm{H} \alpha$ can be found from the FORS spectra, yielding $S F R_{\mathrm{H} \alpha}=0.065 \pm 0.005 M_{\odot} \mathrm{yr}^{-1}$. Due to slit losses the true $\mathrm{H} \alpha$ flux is likely to be higher, and the $S F R_{\mathrm{H} \alpha}$ can be interpreted as a lower limit.

Radio and submillimetre observations do not suffer from dust extinction. The radio continuum flux of a normal galaxy (i.e. non-AGN hosting) is thought to be formed by synchrotron emission by accelerated electrons in supernova remnants and by free-free emission from H II regions (Condon 1992). It is expected that the radio continuum flux is a particularly good tracer of the recent SFR, due to the short expected lifetime of the supernova remnants, which is $\$ 10^{8}$ yr. We use the method described by Vreeswijk et al. (2001) and Berger et al. (2003) to calculate an upper limit to the full star formation rate (i.e. not influenced by any form of dust extinction). We use the deepest $6 \mathrm{~cm}$ (4.9 GHz) Westerbork Synthesis Radio Telescope (WSRT) flux limit, i.e. when the initial radio afterglow has faded beyond detection limit, and find a $3 \sigma$ limit of $72 \mu \mathrm{Jy}$ (formal flux measurement $8 \pm 24 \mu \mathrm{Jy}$, Kaneko et al. 2006) at a $12 \mathrm{~h}$ full synthesis on April 12006 . This leads to a $3 \sigma$ SFR upper limit of $S F R_{\text {radio }}<0.15 M_{\odot} \mathrm{yr}^{-1}$, which excludes a large amount of obscured star formation when compared to $S F R_{\mathrm{H} \alpha}$. However, we note that despite many similarities, GRB hosts studied to date are a diverse population: a handful of hosts show clear indications of much higher star formation rates than seen from optical indicators (comparable to the submillimetre galaxies at several hundred $M_{\odot} \mathrm{yr}^{-1}$ ) from their submm fluxes (Berger et al. 2003; Barnard et al. 2003) and in one case (GRB 980703 at $z=0.97$,
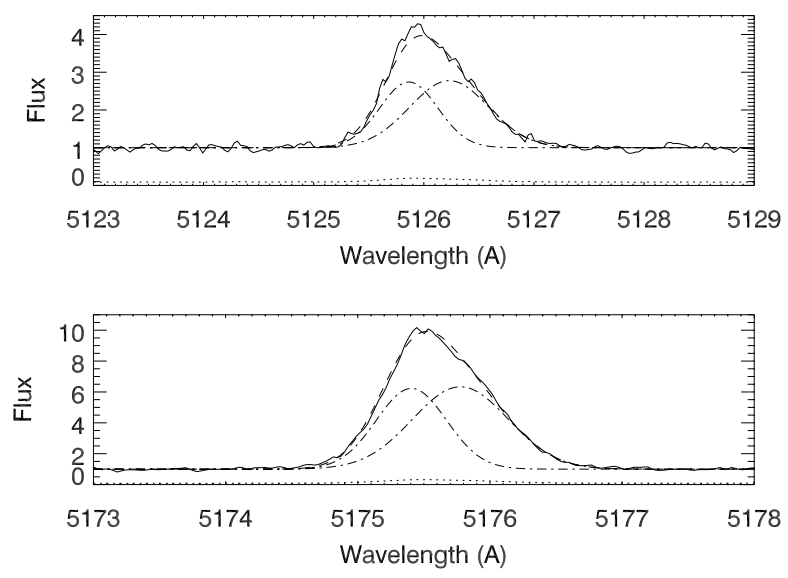

Fig. 4. The [O III] $\lambda 4959$ (top) and $\lambda 5008$ (bottom) emission lines. The continuum is normalized. The error spectrum is shown by a dotted line. The lines are simultaneously fit using fixed flux ratios between $\lambda 4959$ and $\lambda 5008$ components of 1:3. The two seperate Gaussian components are shown with dash-dotted lines, and the total emission with a dashed line.

Berger et al. 2003) its radio flux. However, the radio derived SFRs may systematically overestimate star formation through a non-negligible contribution from AGN activity to the total radio continuum flux.

One of the properties that sets GRB hosts apart from field galaxies is the specific star formation rate (SSFR): the starformation rate per unit mass. This quantity is shown to be high in GRB hosts (e.g. Christensen et al. 2004; Courty et al. 2004). Sollerman et al. (2006) find that the host of GRB 060218 has $L=0.008 L_{B}^{*}$, implying a SSFR of $\sim 8 M_{\odot} \mathrm{yr}^{-1}\left(L / L_{*}\right)^{-1}$. This value is comparable to the SSFRs for a sample of GRB hosts determined through SED fitting by Christensen et al. (2004). Given the multi band SDSS photometry of the host, a low stellar mass is expected. After modeling the SED of the host, Savaglio, Glazebrook \& Le Borgne (in preparation) find $M_{*}=$ $10^{7.2 \pm 0.3} M_{\odot}$. The measured metallicity and stellar mass therefore place this galaxy on the mass-metallicity relation found recently in local dwarf galaxies by Lee et al. (2006a).

\section{Discrete velocity components in emission and absorption}

\subsection{Emission line profiles}

The resolution of the UVES spectrum is sufficient to search for structure in the emission line profiles. Figure 4 shows that the brightest emission lines of [O III] $\lambda \lambda 4959,5008$ significantly deviate from a single Gaussian line profile, and are skewed towards the blue. We can rule out an instrumental effect as no such effect was seen in the arc or sky line profiles. Due to a lower signal to noise, we are not able to verify quantitatively whether other emission lines share the same line profile. We fit Gaussian components to the lines, where the number of components and the position and width of the components are free parameters. The [O III] $\lambda \lambda 4959,5008$ lines are fit simultaneously with a fixed flux ratio between the $\lambda 4959$ and $\lambda 5008$ components of $1: 3$. We use both a fit by eye with IRAF splot and a quantitative deblending using VPFIT $^{1}$. Results agree, and we find a good fit (reduced $\chi^{2} \sim 0.72$ ) using two Gaussian components, shown in Fig. 4, where the components are bluewards and redwards of

${ }^{1}$ See http://www.ast.cam.ac.uk/ rfc/vpfit.html 


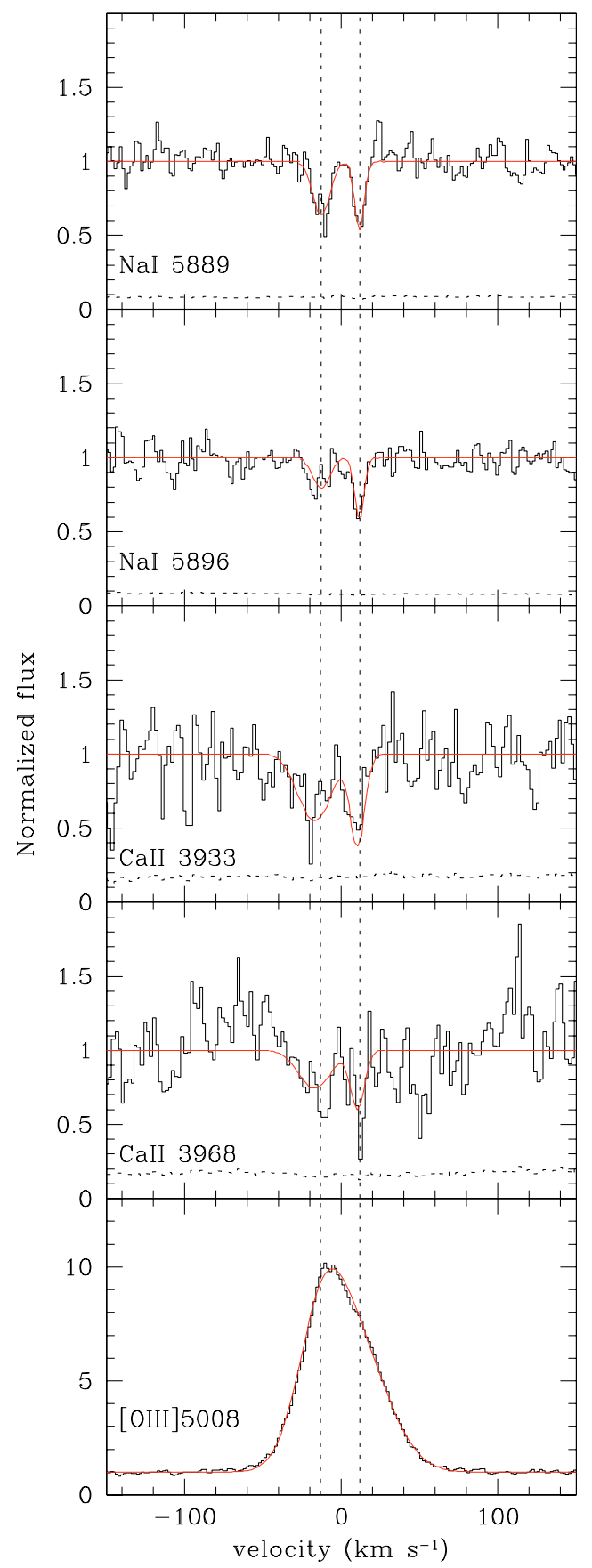

Fig. 5. The NaI and Ca II absorption lines detected in GRB 060218. The two velocity components are marked by the dashed lines (more precisely, these mark the position of the two Na I absorption lines). The smooth line is the result of the best fit Voigt profile (reported in Table 2). The bottom panel shows the [OIII] $\lambda 5008$ emission line for comparison. The dotted spectrum is the noise.

the average host galaxy redshift. For the red component we find a $F W H M$ of $49 \pm 5 \mathrm{~km} \mathrm{~s}^{-1}$ and redshift $0.033453 \pm 0.000019$; and for the blue component a FWHM of $35 \pm 3 \mathrm{~km} \mathrm{~s}^{-1}$ and redshift $0.033379 \pm 0.000005$. This gives $\Delta z=7.4 \pm 2.4 \times 10^{-5}$, or $\sim 21.6 \mathrm{~km} \mathrm{~s}^{-1}$ velocity separation. These values are similar to those found from spatially resolved high resolution spectroscopy of emission line regions (i.e. the 30 Doradus nebula, see Melnick et al. 1999, for a H $\alpha$ study). Arsenault \& Roy (1986) show that $\approx 66 \%$ of all giant extragalactic $\mathrm{H}$ II regions show symmetrical $\mathrm{H} \alpha$ lines in their integrated spectra; the remaining profiles show asymmetries similar to the ones in the host of GRB 060218. When asymmetric line profiles are observed (i.e. in high resolution studies of galactic and extragalactic H II regions) the explanation of their profiles is usually only possible by using a high spatial resolution and correlation of the spectra with images. As an example, the integrated $\mathrm{H} \alpha$ line profile of 30 Doradus shows a broad and narrow Gaussian component (Melnick et al. 1999), whose central wavelengths coincide, while spectra taken at multiple positions in the nebula show a large variety of line profiles and Gaussian components, which generally can be associated with filaments in the nebula. In the case of this host galaxy, we observe the integrated profile over $7 \mathrm{kpc}$.

There are several possible explanations for the occurence of two lines, with the broader one redshifted with respect to the blue component. One possibility is that we see two separate star forming regions in the host. An alternative explanation is that the components are caused by an expanding shell (bubble) around the starforming region; or by infalling gas onto the $\mathrm{H}$ II region (e.g. ionised gas ejected by perhaps SN shocks or stellar winds, that falls back). Neither of the last two scenarios seem plausible: an expanding shell is not likely to have the measured velocity width; and the infalling gas would have to be very highly excited to generate the required [O III] luminosity, which makes it difficult to have co-exisiting $\mathrm{Na}$ I (see Sect. 4.2). We tentatively interpret the two components as arising from two different starforming regions in the host. High resolution imaging would be necessary to confirm this. There are several GRB hosts where HST images resolve the host into multiple starforming regions with similar brightnesses (see e.g. Fruchter et al. 2006). We note that this is the first identification of resolved emission line components in a GRB host galaxy spectrum.

\subsection{Absorption lines in the circumburst medium}

In the UVES spectrum we detect absorption lines at $\sim 4070 \AA$, $\sim 4100 \AA$ and $\sim 6090 \AA$, associated with Ca II $\lambda \lambda 3934,3969$ and $\mathrm{NaI} \lambda \lambda 5891,5897$ in the foreground gas of the GRB/SN, and within the host galaxy. Figure 5 shows at least two discrete velocity components, separated in velocity by $\sim 24 \mathrm{~km} \mathrm{~s}^{-1}$ (systems 1 and 2 in Table 2; see also Guenther et al. 2006). The Ti II absorption lines are not detected because they are in a very noisy region of the spectrum $(\lambda<3500 \AA)$.

The observed lines have been fitted with Voigt profiles using the MIDAS package FITLYMAN (Fontana \& Ballester 1995). Figure 5 shows the fitting results, and column densities and Doppler parameters are listed in Table 2 . The lines are barely resolved, i.e. the line widths are at the level of the UVES spectral resolution $F W H M \simeq 6.5 \mathrm{~km} \mathrm{~s}^{-1}$.

The measured $F W H M$ corresponds to an instrumental PSF of $3.9 \mathrm{~km} \mathrm{~s}^{-1}$, if expressed in terms of Doppler parameter, which can be translated into an upper limit on the gas temperature (derived from the lightest element $\mathrm{Na}$, assuming thermal broadening) of $T \lessgtr 2 \times 10^{4} \mathrm{~K}$. Narrow metal lines are often detected in GRB afterglows when high resolution spectra are acquired (see for instance Chen et al. 2006).

There are indications that $\mathrm{Na}$ I and $\mathrm{Ca}$ II are not tracing each other. The positions of the two Ca II components are shifted by a few $\mathrm{km} \mathrm{s}^{-1}$ with respect to $\mathrm{Na}$, which could be consistent with uncertainties on the fitted parameters (more severe for the Ca II doublet). However, the line broadenings and $\mathrm{Ca}$ II/Na I ratios are different in the two components. We note that in the Galactic ISM Ca II and Na I are found in regions with different physical conditions. The former is found to be generally broader than 
Table 2. Absorption lines in the UVES spectrum of GRB 060218.

\begin{tabular}{clcccccc}
\hline \hline System & Line & $\begin{array}{c}\lambda_{\mathrm{o}} \\
(\AA)\end{array}$ & $z$ & $\begin{array}{c}W_{\mathrm{o}} \\
(\AA)\end{array}$ & $\begin{array}{c}\log N \\
{\left[\mathrm{~cm}^{-2}\right]}\end{array}$ & $\begin{array}{c}b \\
\left(\mathrm{~km} \mathrm{~s}^{-1}\right)\end{array}$ & $\log$ (Na I/Ca II) \\
\hline \multirow{2}{*}{1} & Na I $\lambda 5891$ & 6088.23 & 0.033378 & $0.091 \pm 0.008$ & $11.79 \pm 0.04$ & $6.3 \pm 1.0$ & $-0.54 \pm 0.08$ \\
& Na I $\lambda 5897$ & 6094.41 & & $0.049 \pm 0.007$ & & & \\
& Ca II $\lambda 3934$ & 4066.05 & 0.033363 & $0.093 \pm 0.029$ & $12.33 \pm 0.07$ & $13.3 \pm 3.2$ & \\
& Ca II $\lambda 3969$ & 4102.03 & & $0.064 \pm 0.028$ & & & \\
\hline 2 & Na I $\lambda 5891$ & 6088.73 & 0.033462 & $0.071 \pm 0.006$ & $12.22 \pm 0.22$ & $1.1 \pm 0.2$ & $0.01 \pm 0.23$ \\
& Na I $\lambda 5897$ & 6094.90 & & $0.064 \pm 0.006$ & & & \\
& Ca II $\lambda 3934$ & 4066.42 & 0.033458 & $0.075 \pm 0.025$ & $12.21 \pm 0.08$ & $4.6 \pm 1.8$ & \\
& Ca II $\lambda 3969$ & 4102.40 & & $0.054 \pm 0.029$ & & & \\
\hline Galaxy & Na I $\lambda 5891$ & 5891.75 & 0.0000285 & 0.33 & $12.68 \pm 0.02$ & $6.4 \pm 0.2$ & $0.27 \pm 0.06$ \\
& Na I $\lambda 5897$ & 5897.73 & & 0.25 & & & \\
& Ca II $\lambda 3934$ & 3934.93 & 0.0000381 & 0.13 & $12.41 \pm 0.06$ & $(5)$ & \\
& Ca II $\lambda 3969$ & 3969.74 & & 0.08 & & & \\
\hline
\end{tabular}

Table 3. Table of emission lines used in this paper. Fluxes and $E W \mathrm{~s}$ are as observed. Fluxes from the FORS spectrum are taken from Pian et al. (2006).

\begin{tabular}{|c|c|c|}
\hline \multicolumn{3}{|c|}{ UVES spectrum } \\
\hline \multirow[t]{2}{*}{ ID } & $E W$ & Flux \\
\hline & $(\AA)$ & $\left(\times 10^{-17} \mathrm{erg} \mathrm{s}^{-1} \mathrm{~cm}^{-2}\right)$ \\
\hline [O II] $\lambda 3727$ & $1.59 \pm 0.09$ & $75.23 \pm 3.42$ \\
\hline [O II] $\lambda 3729$ & $2.62 \pm 0.12$ & $121.1 \pm 4.0$ \\
\hline [Ne III] $\lambda 3869$ & $0.75 \pm 0.08$ & $34.06 \pm 3.51$ \\
\hline [Ne III] $\lambda 3969$ & $0.26 \pm 0.06$ & $11.80 \pm 2.64$ \\
\hline $\mathrm{H} \beta \lambda 4862$ & $2.17 \pm 0.06$ & $92.92 \pm 1.93$ \\
\hline $\mathrm{H} \gamma \lambda 4341$ & $0.90 \pm 0.07$ & $47.99 \pm 3.46$ \\
\hline $\mathrm{H} \delta \lambda 4102$ & $0.61 \pm 0.07$ & $27.83 \pm 3.04$ \\
\hline $\mathrm{H} \epsilon \lambda 3971$ & $0.29 \pm 0.07$ & $13.03 \pm 3.02$ \\
\hline H8 $\lambda 3889$ & $0.27 \pm 0.07$ & $12.25 \pm 2.90$ \\
\hline H9 $\lambda 3835$ & $0.18 \pm 0.07$ & $7.65 \pm 2.92$ \\
\hline [O ІІІ] $\lambda 4960$ & $2.88 \pm 0.06$ & $139.0 \pm 2.08$ \\
\hline [O III] $\lambda 5008$ & $8.34 \pm 0.09$ & $426.2 \pm 2.48$ \\
\hline Не г $\lambda 5877$ & $0.24 \pm 0.04$ & $9.55 \pm 1.17$ \\
\hline \multicolumn{3}{|c|}{ FORS spectrum } \\
\hline \multirow[t]{2}{*}{ ID } & & Flux \\
\hline & & $\left(\times 10^{-17} \mathrm{erg} \mathrm{s}^{-1} \mathrm{~cm}^{-2}\right)$ \\
\hline \multirow{2}{*}{\multicolumn{2}{|c|}{$\begin{array}{l}\mathrm{O} \text { II] } \lambda 3727,3729 \\
\text { (Doublet unresolved }\end{array}$}} & $190 \pm 50$ \\
\hline & (Doublet unresolved) & \\
\hline \multicolumn{2}{|l|}{$[\mathrm{Ne}$ III $] \lambda 3869$} & $31 \pm 5$ \\
\hline \multicolumn{2}{|l|}{ [Ne III] $\lambda 3969$} & $18 \pm 3$ \\
\hline \multicolumn{2}{|l|}{$\mathrm{H} \gamma \lambda 4341$} & $37 \pm 6$ \\
\hline \multicolumn{2}{|l|}{ [O III] $\lambda 4364$} & $21 \pm 4$ \\
\hline \multicolumn{2}{|l|}{$\mathrm{H} \beta \lambda 4862$} & $101 \pm 4$ \\
\hline \multicolumn{2}{|l|}{ [O III] $\lambda 4960$} & $150 \pm 7$ \\
\hline \multicolumn{2}{|l|}{ [O III] $\lambda 5008$} & $460 \pm 10$ \\
\hline \multicolumn{2}{|l|}{ Не г $\lambda 5877$} & $5.5 \pm 1.0$ \\
\hline \multicolumn{2}{|l|}{ Н $\alpha \lambda 6563$} & $315 \pm 25$ \\
\hline \multicolumn{2}{|l|}{ [N II] $\lambda 6584$} & $19 \pm 2$ \\
\hline \multicolumn{2}{|l|}{ [S II] $\lambda 6717$} & $14 \pm 2$ \\
\hline \multicolumn{2}{|l|}{ [S II] $\lambda 6731$} & $14 \pm 2$ \\
\hline
\end{tabular}

the latter, indicating that it traces warmer, more turbulent, and/or larger gas clouds (Welty et al. 1996).

Remarkably, the positions of the two absorption systems are consistent with the redshifts of the two emission-line components in the HII regions derived independently (see Sect. 3.5 and the lower panel of Fig. 5). The difference in redshift between emission and absorption is $<1 \mathrm{~km} \mathrm{~s}^{-1}$ and $\sim 2 \mathrm{~km} \mathrm{~s}^{-1}$ for the blueshifted and redshifted systems, respectively. The relative velocity for the two emission components is $21 \mathrm{~km} \mathrm{~s}^{-1}$, close to the $24 \mathrm{~km} \mathrm{~s}^{-1}$ measured from the absorption systems. However, the broader component in absorption is at the lowest redshift, whereas the opposite is true for the emission.

The Ca II column density was measured in another two GRB afterglows (Savaglio \& Fall 2004; Savaglio 2006) with a total column density of nearly $10^{14} \mathrm{~cm}^{-2}$ in each of them. Na I absorption in GRBs is reported here for the first time, basically due to a lack of suitable data in past GRB observations ( $\mathrm{Na}$ I is redshifted into the NIR for $z>0.7$ ). The Na I and $\mathrm{Ca}$ II abundances have been studied in the Galaxy and LMC (Hunter et al. 2006; Vladilo et al. 1993). Beyond the Local Group, $\mathrm{Na}$ I and $\mathrm{Ca}$ II have been detected in 2 damped Lyman- $\alpha$ systems (DLAs) in QSO spectra, at $z=1.062$ and 1.181 (Petitjean et al. 2000; Kondo et al. 2006). Other QSO absorption line studies report upper limits for Na I (Boksenberg et al. 1978, 1980). GRB 060218 is the third source outside the Local Group where NaI is measured ${ }^{2}$.

It is rather complicated to interpret the detection of $\mathrm{Na} I$ and Ca II in GRB 060218 in terms of relative abundances. The ionization potentials of the two ions are quite different $(5.1 \mathrm{eV}$ and $11.9 \mathrm{eV}$ for Na I and Ca II, respectively). Na II is likely the dominant ion in a neutral-gas environment (the $\mathrm{HI}$ ionization potential is $13.6 \mathrm{eV}$ ), whilst Ca III dominates the calcium species. To derive the total abundance of calcium, a significant ionization correction can therefore be necessary. Moreover, $\mathrm{Ca}$ is an $\alpha$ element, while $\mathrm{Na}$ is not. Hence in low metallicity systems, like GRB 060218, an $\alpha$-element enhancement is expected. Sodium was found to trace iron in stars with metallicities close to Solar, but it can have lower abundances for lower metallicities (Timmes et al. 1995). Our expectations are further complicated by the rather different refractory properties of the two elements: $\mathrm{Na}$ is little depleted on to dust grains, whereas Ca can be $99 \%$ depleted (Welty et al. 1994; Savage \& Sembach 1996). This problem may be somewhat mitigated by the likely negligible dust depletion in the gas, as suggested by the small dust extinction derived in the H II regions of GRB 060218 (see Sect. 3.1).

Nevertheless, we compare $\mathrm{NaI}$ and $\mathrm{Ca}$ II in GRB 060218 with what is typically observed in the ISM of the Milky Way (Fig. 6). The two systems in the host of GRB 060218 lie in the bottom left corner of the distribution. Is the observed $\mathrm{Na}$ and Ca behaving like the neutral gas of the Milky Way? If the absorption lines are arising in a neutral region of the ISM, and if we consider the empirical relation that links $\mathrm{H}$ I to $\mathrm{Na}$ I (derived by Hunter et al. 2006) we would expect an $\mathrm{H} \mathrm{I}$ column density along the GRB sight line of the order of $\log N_{\mathrm{HI}}=20.6 \mathrm{~cm}^{-2}$.

${ }^{2}$ More Na I lines are detected in low $z$ SDSS QSO spectra, but no column density measurements are reported for these systems (V. Wild, private communication). 


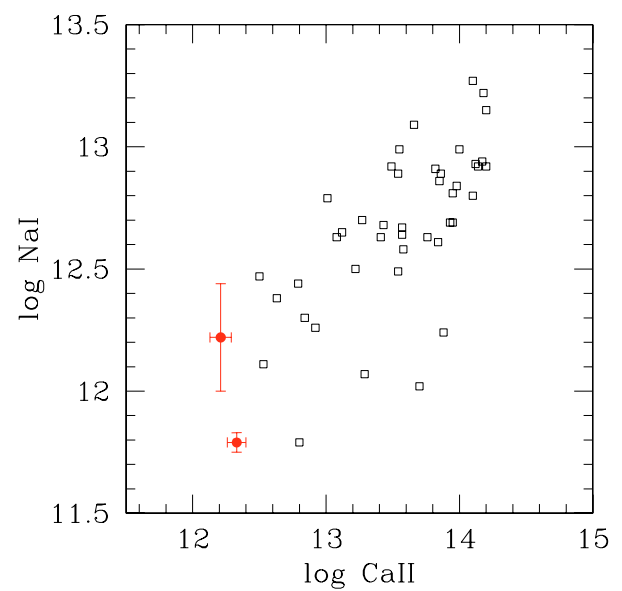

Fig. 6. Na I and Ca II column densities for the two systems in the host of GRB 060218 (filled dots) and lines of sight in the Milky Way (open squares; typical error for these cases is $<0.1 \mathrm{dex}$; Hunter et al. 2006).

However, if the metallicity in the neutral gas is similar to the $\mathrm{H}$ II regions probed by the emission lines, a much lower total H I column density is more likely: $\log N_{\mathrm{H}} \sim 19.4$, suggesting that perhaps the NaI-H I relation found for the Milky Way may not be applicable for the gas around GRB 060218 or that the metallicity in the galaxy is not uniform. An H I column density of the order of $10^{19.4} \mathrm{~cm}^{-2}$ is relatively large, considering that the stellar mass of this GRB host is more than 1000 times smaller than that of the Milky Way. However, a large reservoir of gas is expected given the high SFR per unit stellar mass estimated for the host (see Sect. 3.3).

\section{Secondary metallicity calibrators}

The association of GRB 060218 with a low mass, low metallicity, high excitation host galaxy follows the trend seen from the other nearby GRBs (e.g. Sollerman et al. 2005). In fact, the very low metallicity of the host significantly extends the known metallicities of GRB hosts through emission line spectroscopy. If the redshift of the galaxy had been substantially higher, the [O III] $\lambda 4364$ line would have gone undetected, making a direct determination of the abundance through $T_{\mathrm{e}}$ impossible, and the metallicity inferred from secondary calibrators would have placed the host at significantly higher metallicity (e.g. Mirabal et al. 2006; Modjaz et al. 2006). The sample of GRB host galaxies with a measure of abundances through $T_{\mathrm{e}}$ is limited to just four nearby sources due to the faintness of the [O III] $\lambda 4364$ line, where GRB 020903 has the highest redshift with $z=0.25$. For all other galaxies we are forced to use secondary, empirical methods to calculate metallicity. The most common is the R23 calibrator (see e.g. Kobulnicky $\&$ Kewley 2004), which uses the bright [O III], H $\beta$ and [O II] lines. This method produces a degenerate metallicity solution, which can be broken through other emission lines (e.g. [N II] and $\mathrm{H} \alpha$ ), but in many cases these other lines are not available (due to e.g. redshift or insufficient $\mathrm{S} / \mathrm{N}$ ). The R23 method is calibrated through photo-ionization models, which have limitations at the low and high metallicity ends. It has been shown that R23 metallicities have an offset with respect to $T_{\mathrm{e}}$ metallicities for metallicities close to Solar (e.g. Bresolin et al. 2004). Modjaz et al. (2006) use the R23 method to find $12+\log (\mathrm{O} / \mathrm{H})=8.0 \pm$ 0.1 for the host of GRB 060218. An alternative method that is calibrated on a sample of H II regions with $T_{\mathrm{e}}$ determined metallicities, is the ratio of the nebular [O III] and [N II] lines (e.g. Pettini \& Pagel 2004). The N2 index $(N 2=$ $\left.\log \left(\left[\mathrm{N}_{\mathrm{II}}\right] \lambda 6583 / \mathrm{H} \alpha\right)\right)$ has been proposed particularly for low metallicity galaxies. However, for GRB 060218, the N2 calibration overestimates the metallicity of the host by more than a factor of 3 , yielding $12+\log (\mathrm{O} / \mathrm{H}) \sim 8.2$, but the scatter in this relation is large $(90 \%$ of the fitted data in Pettini \& Pagel 2004 falls within a range $\log (\mathrm{O} / \mathrm{H})= \pm 0.4)$. The $O 3 N 2=\log (([\mathrm{O} \mathrm{III}] \lambda 5008 / \mathrm{H} \beta) /([\mathrm{N} \mathrm{II}] \lambda 6583 / \mathrm{H} \alpha))$ ratio has significantly less scatter but above $03 N 2 \gtrsim 1.9$ this indicator breaks down and cannot be reliably used (Pettini \& Pagel 2004). Our host has $O 3 N 2=1.88 \pm 0.11$, which may explain the overestimate of the metallicity through this indicator of $12+\log (\mathrm{O} / \mathrm{H}) \approx 8.1$.

Concluding, we can state that an analysis using a variety of different secondary methods (e.g. R23, O3N2) would not have found the true metallicity, but may still have identified this host as a very low metallicity candidate.

\section{Massive stars and progenitors}

Long GRB progenitors are likely massive Wolf-Rayet (WR) stars. We can gain a greater understanding of the evolutionary paths of such massive stars towards GRBs by detecting WR populations within GRB host galaxies. A valuable diagnostic on the massive star population is the He II $\lambda 4686$ line, which appears as a broad line and/or as a nebular line in some H II galaxies (Schaerer et al. 1999), and is a direct sign of (unusually) high excitation levels caused by the presence of WR stars (especially WC- and WO-type WR stars). Together with,

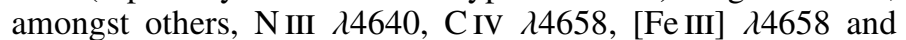
[Ar IV] $\lambda 4711$ lines, this line forms the so-called blue WR bump (from 4650-4700 $\AA$ ) in low resolution spectra, which is often accompanied by a C IV $\lambda 5808$ line (the red bump). In a recent deep spectroscopic search in nearby GRB host galaxies, Hammer et al. (2006) have detected the He II line and accompanying WR bump in the spectra of the hosts of GRB 980425 , 020903 and 031203 . From the relative intensities of the $\mathrm{H} \beta$ and He II $\lambda 4686$ lines or WR bump (measured flux ratios $\mathrm{H} \beta / \mathrm{He}$ II $\lambda 4686$ generally range from $\sim 0.01-0.02$ ) one can estimate the ratio of WR to O stars.

In Crowther \& Hadfield (2005) the effect of metallicity on the derived $\mathrm{WR} /(\mathrm{WR}+\mathrm{O})$ ratio is investigated, by comparing the fluxes of SMC, LMC and Galactic WR stars with atmosphere models. They find a lower WR line luminosity at decreasing metallicity. In the hosts of GRBs 980425 and 020903, Hammer et al. (2006) find values of WR/O 0.05 and $\sim 0.14-0.2$, respectively. We note that no metallicity correction has been applied to these values, which would increase the number of WR stars as the studied hosts have sub-Galactic metallicity.

These high WR/O ratios are of particular interest as their metallicities are accurately known from $T_{\mathrm{e}}$ analyses: $0.5 Z_{\odot}$ and $0.19 Z_{\odot}$ for GRB 980425 and GRB 020903, respectively (Hammer et al. 2006). At these low metallicities the high measured WR/O ratios may be regarded as evidence for very recent star bursts, but it is difficult to determine the ages of the dominant stellar populations. Optical and near-infrared SED fitting has shown that the dominant stellar populations in GRB hosts are young at typically $\sim 0.1 \mathrm{Gyr}$ (Christensen et al. 2004). In the case of the host of GRB 980425 the entire galaxy is well fitted with a continuous star formation history (Sollerman et al. 2005), although Hammer et al. (2006) find a young population from the equivalent widths of the emission lines. No strong evidence for a very young starburst is apparent in the host spectrum of GRB 020903, but the merger morphology of the host and the 
emission line EWs may suggest that some recent star formation is present. However, the WR/O ratio of $0.14-0.2$ in the host of GRB 020903 is particularly remarkable, as such abundant production of WR stars at the low metallicity of $0.19 Z_{\odot}$ can only be explained by invoking instant star bursts with peculiar initial mass function within the standard star burst model by Schaerer \& Vacca (1998) (e.g. Fernandes et al. 2004).

Recent stellar evolution models indicate that the effects of rotation may be, in part, responsible for observed high WR/O ratios in galaxies. According to Meynet \& Maeder (2005a), including the effects of rotation significantly enhances the mass loss rates of massive stars during the giant phase compared to the non-rotating case, as the shear instability due to the strong degree of differential rotation between the core and the envelope induces fast chemical mixing. Their models predict a WR/O ratio of about 0.02 at $Z=0.004$ for a constant star formation, and in principle $\mathrm{WR} / \mathrm{O} \sim 0.2$ might be achieved at the given metallicity for instant star bursts even with a standard initial mass function. However, such rotating models predict GRB/SN Ibc ratios that are too high. Their prediction of spin rates of young neutron stars is also inconsistent with observations (Hirschi et al. 2005; see also Heger et al. 2000).

More recent models that include magnetic torques for the angular momentum transport in the star suggest another way to produce WR stars at low metallicity. Although in magnetic models the chemical mixing induced by the shear instability during the giant phase is negligible as the magnetic torque tends to keep the star rigidly rotating, the mixing by meridional circulation can be very fast even on the main sequence (Maeder \& Meynet 2005 b). This may even cause the whole star to become chemically homogeneous if the initial rotational velocity is sufficiently high, especially at sub-solar metallicity (Yoon \& Langer 2005; Woosley \& Heger 2006).

Formation of WR stars can thus be induced not only by mass loss but also by chemical mixing, and Woosley \& Heger (2006) found that some WR stars formed through such chemically homogeneous evolution can actually retain enough angular momentum necessary for GRB production. The predicted GRB/SN ratio through such evolutionary channels also turns out to be consistent with observations, when the observationally derived initial rotational velocity distribution of massive stars by Mokiem et al. (2006) is adopted (Yoon et al. 2006). Interestingly, as stars may be kept rotating rapidly at low metallicity due to lowered mass loss rates (e.g. Vink et al. 2005), this new type of evolution by fast rotational mixing can lead to high WR/O ratios even at very low metallicity (Yoon et al. 2006). It also predicts rather large delay times for WR production from the star formation (i.e., from several $10^{6} \mathrm{yrs}$ to a few $\sim 10^{7} \mathrm{yrs}$; Yoon et al. 2006) compared to those from mass-loss induced WR formation $(<\mathrm{a}$ few $10^{6}$ yrs).

In this regard, an estimate of the WR/O ratio in the host galaxy of GRB 060218 might provide a valuable test case for the new GRB progenitor scenario by Yoon \& Langer (2005) and Woosley \& Heger (2006). That is, if the WR/O ratio in this host turns out to be high despite its very low metallicity, it may support the chemically homogeneous evolution scenario for GRB progenitors and extend the metallicity - WR/O star ratio relation for GRB hosts down to lower metallicity. This relation is well known for the Milky Way, LMC and SMC, and a deviation of GRB hosts away from that trend gives strong input for progenitor modelling.

The high resolution UVES spectrum of GRB 060218 should be able to resolve the components comprising the WR bump, and resolve a He II line into a broad and a nebular component.
However, no nebular He II line or WR bump has been significantly detected. We measure the flux upper limit on the WR bump in the UVES spectrum by summing the flux in the WR bump wavelength region (restframe 4650-4686 A). Following the method of Schaerer \& Vacca (1998) we set an upper limit of $\mathrm{WR} /(\mathrm{WR}+\mathrm{O}) \lesssim 0.4$, which is not a constraining limit, owing to the fact that the SN outshines the possible He II line. To reliably detect the WR bump in this host we need to detect the host continuum with reasonable $\mathrm{S} / \mathrm{N}$, which means the supernova has to fade below this level before more sensitive searches are feasible.

\section{Conclusions}

We present a VLT UVES high resolution spectrum of SN 2006aj, associated with the nearby GRB 060218 at heliocentric redshift $z=0.03342(2)$. We use the emission lines of the UVES spectrum as well as the line measurements from our FORS spectroscopic campaign to derive properties of the host. We find that the electron density is low, and the electron temperature is high, $T_{\mathrm{e}}\left(\mathrm{O}^{2+}\right)=2.48_{-0.3}^{+0.5} \times 10^{4} \mathrm{~K}$, as shown in Table 1 . We find a low host metallicity of $12+\log (\mathrm{O} / \mathrm{H})=7.54_{-0.10}^{+0.17}$, placing it among the most metal deficient subset of emission-line galaxies. It is also the lowest metallicity found so far for a GRB host from an emission line analysis. The metallicity we find lies considerably below the values derived using secondary calibrators, e.g. the metallicity $12+\log (\mathrm{O} / \mathrm{H})=8.0$ as derived from the R23 calibrator (e.g. Modjaz et al. 2006). The mass of the galaxy is low, and matches what is expected from the mass-metallicity relation for dwarf galaxies. We measure a relatively high value for $\log (\mathrm{N} / \mathrm{O})$ with respect to the metallicity, which is also seen in a few other GRB hosts. As our uncertainty on $\log (\mathrm{N} / \mathrm{O})$ is relatively high, deeper spectroscopy is needed to confirm this overabundance.

The bright emission lines show strong evidence for asymmetry, and a single Gaussian provides a poor fit to the profiles of the bright [O III] emission lines. A two Gaussian model provides a satisfactory fit with the two components separated by $\sim 22 \mathrm{~km} \mathrm{~s}^{-1}$. We find the same two velocity components in absorption through the $\mathrm{Ca}$ II and $\mathrm{Na}$ I absorption lines in the host. We tentatively interpret these two velocity components to be due to two star forming regions in the host galaxy. However, to unravel their true identity, high spatial resolution imaging is needed.

The dust content of the galaxy is low, based on the Balmer line decrement. This is also evident from the low limit on the obscured star formation rate we set through a $3 \sigma$ upper limit on the flux at $6 \mathrm{~cm}$ of $S F R_{\text {radio }}<0.15 M_{\odot} \mathrm{yr}^{-1}$, compared to the optical star-formation rate $S F R_{\mathrm{H} \alpha}=0.065 \pm 0.005 M_{\odot} \mathrm{yr}^{-1}$.

This host galaxy is an interesting target for future spectroscopy targeted at the WR bump, as the low metallicity of the host will significantly extend the present sample of GRB hosts with known WR star content and metallicity. We show that a measure of these two quantities for a sample of GRB hosts may provide further insight into the nature of GRB progenitors.

The absolute magnitude of the host $\left(M_{B}=-15.9\right.$, e.g. Sollerman et al. 2006) is such that this galaxy would not have been detected in any survey at a redshift of $z \sim 1$, let alone at the mean Swift GRB redshift of $z \sim 2.8$ (Jakobsson et al. 2006), which makes this host an important object to study in the context of larger redshift GRB hosts.

Acknowledgements. We thank the observers and Paranal staff for performing the reported observations at ESO VLT. We are very grateful to R. B. C. Henry, H. Lee and N. Tanvir for helpful discussions. We thank the anonymous referee for helpful comments. K.W. thanks NWO for support under grant 639.043.302. The 
Dark Cosmology Centre is funded by the Danish National Research Foundation. S.C.Y. is supported by the VENI grant (639.041.406) of The Netherlands Organization for Scientific Research (NWO). The authors acknowledge benefits from collaboration within the EU FP5 Research Training Network "Gamma-Ray Bursts: An Enigma and a Tool" (HPRN-CT-2002-00294).

\section{References}

Allende Prieto, C., Lambert, D. L., \& Asplund, M. 2001, ApJ, 556, L63 Arsenault, R., \& Roy, J.-R. 1986, AJ, 92, 567

Barnard, V. E., Blain, A. W., Tanvir, N. R., et al. 2003, MNRAS, 338, 1 Berger, E., Cowie, L. L., Kulkarni, S. R., et al. 2003, ApJ, 588, 99

Bloom, J. S., Kulkarni, S. K., \& Djorgovski, S. G. 2002, AJ, 123, 1111

Boksenberg, A., Carswell, R. F., Smith, M. G., et al. 1978, MNRAS, 184, 773 Boksenberg, A., Danziger, I. J., Fosbury, R. A. E., et al. 1980, ApJ, 242, 145

Bresolin, F., Garnett, D. R., \& Kennicutt, R. C. Jr. 2004, ApJ, 615, 228

Campana, S., Mangano, V., Blustin, A. J., et al. 2006 [arXiv:astro-ph/0603279]

Cardelli, J. A., Clayton, G. C., \& Mathis, J. S. 1989, ApJ, 345, 245

Chary, R., Becklin, E. E., \& Armus, L. 2002, ApJ, 566, 229

Chen, H.-W., Prochaska, J. X., Bloom, et al. 2005, ApJ, 634, 25

Chen, H.-W., Prochaska, J. X., \& Bloom, J. S. 2006, Proceedings of the 16th Annual October Astrophysics Conference in Maryland, Gamma Ray Bursts in the Swift Era, ed. S. Holt, N. Gehrels, \& J. Nousek [arXiv: astro-ph/0602144]

Christensen, L., Hjorth, J. \& Gorosabel, J. 2004, A\&A, 425, 913

Condon, J. J. 1992, ARA\&A, 30, 575

Courty, S., Björnsson, G., \& Gudmundsson, E. H. 2004, MNRAS, 354, 581

Crowther, P. A., \& Hadfield, L. J. 2005, A\&A, 449, 711

Dekker, H., D’Odorico, S., Kaufer, A., et al. 2000, SPIE, 4008, 534

Ellison, S. L., \& Kewley, L. J. 2006, Proceedings The Fabulous Destiny of Galaxies; Bridging the Past and Present [arXiv:astro-ph/0508627]

Fernandes, I. F., de Carvalho, R., Contini, T., et al. 2004, MNRAS, 355, 728

Fontana, A., \& Ballester, P. 1995, The ESO Messenger, 80, 37

Fruchter, A. S., Thorsett, S. E., Metzger, M. R., et al. 1999, ApJ, 519, L13

Fruchter, A. S., Levan, A. J., Strolger, L., et al. 2006, Nature, 441, 463

Fukugita, M., Shimasaku, K., \& Ichikawa, T. 1995, PASP, 107, 945

Guenther, E. W., Klose, S., Vreeswijk, P. M., et al. 2006, GCN Circulars, 4863 Hammer, F., Flores, H., Schaerer, D., et al. 2006, A\&A, 454, 103

Heger, A., Langer, N., \& Woosley, S. E. 2000, ApJ, 528, 368

Hirschi, R., Meynet, G., \& Maeder, A. 2005, A\&A, 443, 581

Hjorth, J., Sollerman, J., Møller, P., et al. 2003, Nature, 423, 847

Hunter, I., Smoker, J. V., Keenan, F. P., et al. 2006, MNRAS, 367, 1478

Izotov, Y. I., Thuan, T. X., \& Lipovetsky, V. A. 1994, ApJ, 435, 647

Izotov, Y. I., Chaffee, F. H., Foltz, C. B., et al. 1999, ApJ, 527, 757

Izotov, Y. I., Stasińska, G., Meynet, G., et al. 2006a, A\&A, 448, 955

Izotov, Y. I., Papaderos, P., Guseva, N. G., et al. 2006b, A\&A, 454, 137

Jakobsson, P., Björnsson, G., Fynbo, J. P. U., et al. 2005, MNRAS, 362, 245

Kaneko, Y., Ramirez-Ruiz, E., Granot, J., et al. 2006

[arXiv: astro-ph/0607110]

Kauffmann, G., Heckman, T. M., Tremonti, C., et al. 2003, MNRAS, 346, 1055
Kawai, N., Kosugi G., Aoki, K., et al. 2006, Nature, 440, 184

Kennicutt, R. C. 1998, ARA\&A, 36, 189

Kewley, L. J., Brown, W. R., Geller, M. J., et al. 2006

[arXiv: astro-ph/0609246]

Kobulnicky, H. A., \& Kewley, L. J. 2004, ApJ, 617, 240

Kondo, S., Kobayashi, N., Minowa, Y., et al. 2006, ApJ, 643, 667

Langer, N., \& Norman, C. A. 2006 ApJ, 638, L63

Lodders, K. 2003, ApJ, 591, 1220

Lee, H., Skillman, E. D., Cannon, J. M., et al. 2006a, ApJ, in press [arXiv: astro-ph/0605036]

Lee, H., Skillman, E. D., \& Venn, K. A. 2006b, ApJ, 642, 813

Le Floc'h, E., Duc, P.-A., Mirabel, I. F., et al. 2003, A\&A, 400, 499

Lopez, S., \& Ellison, S. L. 2003, A\&A, 403, 57033

Mazzali, P. A., Deng, J., Nomoto, K., et al. 2006, Nature, 442, 1018

Melnick, J., Tenorio-Tagle, G., \& Terlevich, R. 1999, MNRAS, 302, 677

Meynet, G., \& Maeder, A. 2005a, A\&A, 429, 581

Meynet, G., \& Maeder, A. 2005b, A\&A, 440, 104

Mirabal, N., \& Halpern, J. P. 2006, GCN Circulars, 4792

Mirabal, N., Halpern, J. P., An, D., et al. 2006, ApJ, 643, 99

Modjaz, M., Stanek, K. Z., Garnavich, P. M., et al. 2006, ApJ, 645, 21

Mokiem, M., de Koter, A., Evans, C., et al. 2006, A\&A, 456, 1131

Moustakas, J., Kennicutt, R. C., \& Tremonti, C. A. 2006, ApJ, 642, 775

Osterbrock, D. E. 1989, Astrophysics of Gaseous Nebulae and Active Galactic Nuclei (Mill Valley: University Science Books)

Pagel, B. E. J., Simonson, E. A., Terlevich, R. J., et al. 1992, MNRAS, 255, 325

Petitjean, P., Aracil, B., Srianand, R., et al. 2000, A\&A, 359, 457

Pettini, M., \& Pagel, B. E. J. 2004, MNRAS, 348, L59

Pian, E., Mazzali, P. A., Masetti, N., et al. 2006, Nature, 442, 1011

Prochaska, J. X., Bloom, J. S., Chen, H., et al. 2004, ApJ, 611, 200

Savage, B. D., \& Sembach, K. R. 1996, ARA\&A, 34, 279

Savaglio, S. 2006, New J. Phys., 8, 195

Savaglio, S., \& Fall, S. M. 2004, ApJ, 614, 293

Schaerer, D., \& Vacca, W. D. 1998, ApJ, 497, 618

Schaerer, D., Contini, T., \& Pindao, M. 1999, A\&AS, 136, 35

Schlegel, D. J., Finkbeiner, D. P., \& Davis, M. 2004, ApJ, 500, 525

Skillman, E. D., Terlevich, R. J., Kennicutt, R. C., Jr., et al. 1994, ApJ, 431, 172

Soderberg, A. M., Kulkarni, S. R., Nakar, E., et al. 2006, Nature, 442, 1014

Sollerman, J., Östlin, G., Fynbo, J. P. U., et al. 2005, NewA, 11, 103

Sollerman, J., Jaunsen, A. O., Fynbo, J. P. U., et al. 2006, A\&A, 454, 503

Stanek, K. Z., Matheson, T., Garnavich, P. M., et al. 2003, ApJ, 591, 17

Stanek, K. Z., Gnedin, O. Y., Beacom, J. F., et al. 2006 [arXiv: astro-ph/0604113]

Starling, R. L. C., Vreeswijk, P. M., Ellison, S. L., et al. 2005, A\&A, 442, 21

Stasińska, \& Izotov 2003, A\&A, 397, 71

Timmes, F. X., Woosley, S. E., \& Weaver, T. A. 1995, ApJS, 98, 617

Van Dokkum, P. G. 2001, PASP, 113, 1420

Vink, J. S., de Koter, A., \& Lamers, H. J. G. L. M. 2001, A\&A, 369, 574

Vladilo, G., Centurion, M., \& Cassola, C. 1993, A\&A, 273, 3

Vreeswijk, P. M., Fender, R. P., Garrett, M. A., et al. 2001, A\&A, 380, L21

Welty, D. E., Hobbs, L. M., Kulkarni, V. P. 1994, ApJ, 436, 152

Welty, D. E., Morton, D. C., \& Hobbs, L. M. 1996, ApJS, 106, 533

Wolf, C., \& Podsiadlowski, P. 2006 [arXiv: astro-ph/0606725]

Woosley, S. E., \& Heger, A. 2006, ApJ, 637, 914

Yoon, S.-C., \& Langer, N. 2005, A\&A, 443, 643

Yoon, S.-C., Langer, N., \& Norman, C. 2006 [arXiv : astro-ph/0606637] 